\title{
Intravenous immunoglobulin treatment in patients with chronic inflammatory demyelinating polyneuropathy
}

\author{
Pieter A van Doorn
}

\begin{abstract}
Intravenous immunoglobulin (IVIg) treatment is shown to be effective in a selected group of patients with a chronic inflammatory demyelinating polyneuropathy (CIDP). The proportion of patients that improve after IVIg treatment varies between studies. Because $40 \%$ of a group of IVIg treated CIDP patients needed intermittent IVIg infusions to maintain their improved clinical condition, it is expected that IVIg is effective, at least in this subgroup of patients. However, the proportion of patients that improve after IVIg is highly dependent on the selection of patients. Patients with signs and symptoms of an active disease and clear involvement of both arms and legs appear to have the highest chance of improvement after IVIg treatment, but additional or prospective studies are needed to verify these criteria. Results obtained from small numbers of patients treated in open studies suggest that CIDP patients with a monoclonal gammopathy may also improve after IVIg treatment. Further studies are required to evaluate the prognostic factors for improvement after IVIg treatment in CIDP patients and to compare the efficacy and safety of IVIg with other long-term treatment regimes. New studies should also focus on the mechanism of IVIg treatment in patients with CIDP.
\end{abstract}

Chronic inflammatory demyelinating polyneuropathy (CIDP), is a symmetrical sensorymotor neuropathy and is considered to be the chronic variety of the Guillain-Barré syndrome (GBS), an acute immune-mediated polyneuropathy. ${ }^{1}$ CIDP and GBS differ mainly in onset, course and prognosis. Patients with GBS generally have progressive weakness of less than four weeks. ${ }^{2}$ In contrast, most patients with CIDP have progressive weakness during a longer period of time, often exceeding two months. It was recently suggested that the term "subacute" inflammatory demyelinating polyneuropathy should be reserved for those patients with a progressive course of four to eight weeks. ${ }^{3}$ According to recent criteria, the minimal duration of progression in CIDP patients should be at least two months. ${ }^{4}$

Patients with CIDP may respond to treatment with corticosteroids and to plasma exchange (PE), as demonstrated in randomised clinical trials. ${ }^{56}$ The chronic course of CIDP in principle offers the possibility of using various kinds of treatment or different dose schedules in single patients. However, the course of CIDP is not completely predictable as these patients can either have spontaneous relapses or a rapid spontaneous clinical improvement which has been observed in some patients even after a course of severe progressive weakness during several months, ${ }^{7}$ and indicates the need for properly designed controlled studies.

\section{IVIg treatment in CIDP}

Long term treatment with corticosteroids can have serious side-effects. ${ }^{8}$ PE is a time consuming procedure, needing specialised equipment and good vascular access. Complications of PE in patients with CIDP are generally not severe. In the early 1980 s, when the results of the corticosteroid and PE trials in patients with CIDP were not available, fresh-frozen plasma (FFP) and later on IVIg was administered to 17 severely disabled CIDP patients. ${ }^{9}$ Improvement was observed in 13 patients $(76 \%)$. Most of these patients needed intermittent treatment and none of them became refractory to IVIg treatment. Since then, several uncontrolled studies claimed that $20-100 \%$ of the CIDP patients may improve after IVIg treatment. The results of the studies concerning more than two patients with CIDP treated with IVIg are shown in the table. ${ }^{9-15}$

These observations were supported by the results of a double-blind placebo controlled cross-over trial in patients with CIDP, who were judged to have responded to IVIg treatment and who needed repeated IVIg infusions to maintain their improved condition. ${ }^{16}$ In this cross-over study all the patients deteriorated after discontinuation of regular IVIg treatment. Thereafter, the patients were randomised to IVIg $(0.4 \mathrm{~g} / \mathrm{kg})$ or placebo (albumin) for five consecutive days of treatment. All the patients responded after IVIg and none responded after placebo treatment. Improvement after IVIg was always observed within one week after the start of treatment. The time-lapse from the end of trial treatment to deterioration was significantly longer after treatment with IVIg (mean 6.4 weeks) than after placebo treatment (mean 1.3 weeks).

In a retrospective study involving 52 patients with CIDP, it was found that 32 


\begin{tabular}{|c|c|c|c|c|c|c|c|c|}
\hline \multirow[b]{2}{*}{ Study } & \multirow[b]{2}{*}{ Type of study } & \multirow[b]{2}{*}{ Co-medication } & \multirow[b]{2}{*}{$\begin{array}{l}\text { IVIg dose } \\
\text { g/kg/d }\end{array}$} & \multirow[b]{2}{*}{$\begin{array}{l}\text { Deterioration } \\
\text { after IVIg }\end{array}$} & \multirow{2}{*}{$\begin{array}{l}\text { Scoring: } \\
\text { difference } \\
\text { in }\end{array}$} & \multirow[b]{2}{*}{$\begin{array}{l}\text { Improvement } \\
\text { onset (days) }\end{array}$} & \multicolumn{2}{|c|}{ Improvement } \\
\hline & & & & & & & $\begin{array}{l}\text { Proportion } \\
\text { of patients }\end{array}$ & $\%$ \\
\hline Vermeulen $^{7}$ & Double blind & No & $0.4 \times 5$ & - & Rankin $\geq 1$ & $14^{\mathrm{a}}$ & $\begin{array}{l}4 / 15 \\
3 / 13\end{array}$ & $\begin{array}{l}27 \\
23\end{array}$ \\
\hline van Doorn ${ }^{16}$ & $\begin{array}{l}\text { Double blind } \\
\text { cross-over }\end{array}$ & No & $\begin{array}{l}0.4 \times 5 \\
\text { placebo }\end{array}$ & 一 & $\begin{array}{l}\text { Rankin } \geq 1 \\
\text { Rankin } \geq 1\end{array}$ & $\begin{array}{l}8^{\mathrm{b}} \\
8^{\mathrm{b}}\end{array}$ & 7/7 (IVIg) & $\begin{array}{l}100 \\
0 / 7\end{array}$ \\
\hline $\begin{array}{l}\text { (placebo) } \\
\text { Vermeulen }\end{array}$ & Open & No & $\begin{array}{l}0 \cdot 1 \text { litre } \\
\text { FFP/kg }\end{array}$ & $\begin{array}{l}9 \text { patients: } \\
3 \text { weeks }\end{array}$ & $\begin{array}{l}\text { disability } \\
\text { grade } \geq 1\end{array}$ & $<8$ & $13 / 17$ & 76 \\
\hline Curro Dossi ${ }^{10}$ & Open & No & $0.4 \times 5$ & $\begin{array}{l}1 \text { patient: } \\
\text { relapse }\end{array}$ & $\begin{array}{l}\text { neuromusc } \\
\text { function }\end{array}$ & $<10$ & $4 / 4$ & 100 \\
\hline Faed $^{11}$ & Open & $\mathrm{p}: 2, \mathrm{az}: 1$ & $0.4 \times 3$ & $\begin{array}{l}4 \text { patients: } \\
1-2 \text { weeks }\end{array}$ & MRC & $2-7$ & $9 / 9$ & 100 \\
\hline Vedanarayanan ${ }^{12}$ & Open & az:2 & $0.4 \times 5$ & $\begin{array}{l}3 \text { patients: } \\
\text { relapse }\end{array}$ & MRC & NM & $4 / 4$ & 100 \\
\hline Cornblath $^{13}$ & Open & p:6,az:2l & $0.3-0.4 \times 4-5$ & $\begin{array}{l}2 \text { patients: } \\
\text { 2-8 weeks }\end{array}$ & $\begin{array}{l}\text { neuromusc } \\
\text { function }\end{array}$ & NM & $3 / 15$ & 20 \\
\hline van Doorn ${ }^{14}$ & Open & No & $0.4 \times 5$ & $\begin{array}{l}21 \text { patients: } \\
2 \text { weeks }\end{array}$ & Rankin $\geq 1$ & $3-8$ & $32 / 52^{\mathrm{c}}$ & 62 \\
\hline Azulay ${ }^{15}$ & Open & No & $0.4 \times 5$ & NM & $\begin{array}{l}\text { neuromusc } \\
\text { function }\end{array}$ & $<14$ & $4 / 8$ & 50 \\
\hline
\end{tabular}

$\mathrm{az}=$ azathioprine, $\mathrm{p}=$ prednisone, $\mathrm{FFP}=$ fresh frozen plasma, $\mathrm{NM}=$ not mentioned.

$\mathrm{a}=$ scoring at day $14 \mathbf{b}^{\mathrm{b}}=$ scoring at day 8 .

$c=32 / 52$ improved; $2 / 32$ only shortlasting; $9 / 32$ one course followed by clinical remission; $21 / 32$ intermittent treatment necessary

patients (62\%) improved after IVIg. Twenty one patients $(40 \%)$ needed intermittent IVIg infusions to maintain clinical improvement suggesting that improvement, at least in these patients, was caused by IVIg treatment. ${ }^{14}$ In two of the 32 patients, there was a short-lasting improvement, and subsequent IVIg infusions had no effect. Eighteen of 30 patients ( $60 \%)$ who needed one or more (intermittent) IVIg infusions to maintain the improved condition, have reached a spontaneous or therapeutically (IVIg) induced complete remission after a mean follow up period of 6.5 years. The following variables were significantly associated with improvement after IVIg treatment: disease duration of less than one year, progression of weakness until treatment, absence of discrepancy in weakness between arms and legs, areflexia of the arms, and reduced nerve conduction velocity of the motor median nerve. ${ }^{14}$

It is surprising that it was not possible to demonstrate the efficacy of IVIg treatment in a double-blind, placebo controlled study in 28 patients with a clinical diagnosis of CIDP who were not treated with IVIg previously. ${ }^{7}$ The reason for this may be the selection of CIDP patients: as many as 10 of 13 patients in the placebo group and only six of 15 patients in the IVIg treatment group fulfilled the five criteria that were associated with improvement after IVIg treatment. ${ }^{14}$ These observations indicate that only a subgroup of patients with a CIDP seem to respond to IVIg treatment. These prognostic factors, however, need to be evaluated in a properly designed prospective study. Another surprising finding was that three patients with a slowly progressive deterioration before the start of trial treatment had a rapid and dramatic clinical improvement within days after the start of placebo treatment. The previously performed cross-over trial, however, showed that CIDP patients responded to IVIg and not to placebo. ${ }^{16}$ It is therefore concluded that we require better criteria to select CIDP patients for treatment with IVIg, and that the follow up must be carefully carried out to prevent continuous prescription of therapy in the case of spontaneous or therapeutically induced clinical remission.

\section{Comparison between treatment with steroids, PE and IVIg}

To date, no controlled study that compares IVIg with corticosteroids or PE treatment has been published. A cross-over study organised by Dyck et al comparing PE with IVIg treatment in 20 CIDP patients has recently been completed in the USA. If the results of this study favour IVIg, this option may be the treatment of choice. However, if the results of the PE treated group are better, this will have to be weighed against the disadvantages of PE treatment: the limited availability for long term treatment in many centres, minor side effects and the requirement of good vascular access in general. Moreover, the costs of treatment are important. IVIg and PE are both expensive treatments. To compare the costs between PE and IVIg, the costs of PE personnel, equipment and other logistics have to be taken into account. The cost for five PE sessions or a IVIg course with in total $2 \mathrm{~g} / \mathrm{kg}$ seems to be more or less equal, not only in our country but also in the United States. ${ }^{17}$ Corticosteroids on the other hand are cheap, but complications can be severe and expensive as well.

Several uncontrolled studies have included CIDP patients who were treated with PE, steroids or other immunosuppressive treatments before IVIg treatment, or alternatively, after a failure of IVIg treatment. Cornblath et al treated 15 CIDP patients with IVIg. ${ }^{13}$ Fourteen patients were treated with PE, steroids or other immunosuppressive treatment before IVIg was started. The indications for IVIg were: failure after one or more therapies (four patients), desire to avoid possible side effects (four patients), and unsatisfactory response to current therapy (seven patients). Six patients were on other therapies at the onset of IVIg treatment. The three patients showing objective improvement after IVIg ini- 
tially had incomplete improvement after prednisone treatment. Some of the patients who did not improve after IVIg or PE, subsequently improved after prednisone treatment. Faed et al reported that prednisone and azathioprine did not alter the pattern of relapses that occurred in a patient needing intermittent IVIg. ${ }^{11}$ In our own series, prednisone treatment resulted in improvement in five of 16 patients $(31 \%)$ who did not respond to IVIg. These observations indicate that some CIDP patients may improve after immunosuppressive treatment, PE or IVIg, while other patients improve only after a particular treatment. This means that it is generally worthwhile to try another treatment modality in an individual patient if either prednisone, PE or IVIg fails.

\section{What treatment first?}

Without a controlled study comparing prednisone, PE and IVIg this is a difficult question to answer. The major drawback of IVIg treatment is its high cost, and frequently this is the reason to chose prednisone as the initial treatment. However, because of the potentially serious side effects of prednisone, especially in children, we start treatment with IVIg as soon as the patient has reached a degree of muscular weakness that significantly interferes with lifestyle or prevents totally independent existence. PE, being roughly as expensive as IVIg, is logistically often difficult to perform if the patient needs long term treatment.

\section{What is the best IVIg dose schedule?}

The best IVIg dose schedule in CIDP patients is not known. Initially, most patients were treated with $0.4 \mathrm{~g} \mathrm{IVIg} / \mathrm{kg} /$ day for five consecutive days. With this dose no major complications were observed in CIDP patients. A CIDP patient may be treated with $1.0 \mathrm{~g} \mathrm{IVIg} / \mathrm{kg} /$ day for two days or even the double dose in a single day. In Kawasaki disease such a rapidly administered total dose appeared to be even more effective. ${ }^{18}$ However, especially in older patients, one needs to be wary of fluid dysbalance and increased plasma viscosity due to an overload of IVIg, ${ }^{19}$ resulting in vascular problems. ${ }^{20}$ We usually treat CIDP patients with $0.4 \mathrm{~g} \mathrm{IVIg} / \mathrm{kg} /$ day for five days and we have observed no major complications in the more than 65 patients treated of whom many needed repeated treatment. If a patient improves after IVIg treatment, we wait and see. When secondary deterioration follows, a $0.4 \mathrm{~g} \mathrm{IVIg} / \mathrm{kg}$ course for one day is recommended. We then put the patient on a regular treatment scheme, that comprises one treatment day every three to four weeks, depending on the severity and rate of deterioration. If this is insufficient, treatment frequency is increased to one day every other week. If the patient reaches a stable condition we gradually reduce the dose instead of increasing the interval between the administered IVIg doses.
Patients with CIDP and a monoclonal protein of undetermined significance

Initially it was suggested that the diagnosis of CIDP can only be made in the absence of a serum monoclonal protein. ${ }^{21}$ However, some patients with the clinical signs and symptoms of a CIDP also have a monoclonal protein of undetermined significance (MGUS). Whether these patients may be diagnosed as having CIDP is presently still a matter of debate. There are several arguments that favour doing so, especially in CIDP patients with an IgG MGUS, older than $45-50$ years of age, since a monoclonal gammopathy is more frequently found in the older population. Patients with a neuropathy and an IgM MGUS, are more difficult to diagnose as CIDP. This is partly due to the fact that roughly half of these patients have serum antibodies against myelin associated glycoprotein (MAG). These antibodies, which are related to the uncompacting of myelin, are not normally found in patients with idiopathic CIDP. Additionally local antibody deposition can be found in nerve biopsies from patients with an IgM MGUS polyneuropathy. This is normally not found in CIDP.

Patients with an IgM MGUS and antiMAG activity often have a slowly progressive, mainly sensory polyneuropathy which is infrequently found in patients with a CIDP. Patients with a chronic neuropathy and an IgM MGUS more frequently have sensory loss and nerve conduction abnormalities compared with patients with an IgG MGUS. ${ }^{22}$ These findings support the view that IgM MGUS neuropathies are different from IgG MGUS neuropathies.

Two recent studies are particularly interesting as 30 of the 100 patients with a clinical diagnosis of CIDP according to the criteria by Dyck $^{21}$ also had a MGUS. ${ }^{23}{ }^{24}$ No clinical or electrophysiological differences were demonstrated between the groups of CIDP patients with an IgG, IgM or IgA MGUS. ${ }^{23}$ Motor conduction studies were similar in CIDP patients with or without a MGUS. However, CIDP patients with a MGUS were older than the patients without a MGUS, ${ }^{23}$ they had on average more frequent sensory loss with less severe weakness, and the course of disease was more indolent. ${ }^{24}$ Most CIDP-MGUS patients $(21 / 26)$ were treated with prednisone or PE. Eleven patients improved, which was significantly less than in the CIDP group without a MGUS. Whether this is due to a possible worse response in the IgM MGUS subgroup is not clear. Several patients who did not respond to one of these treatments were subsequently treated with IVIg or azathioprine. "Most" poor responders after prednisone or PE treatment improved after treatment with a "second or third" modality. One of the CIDP-MGUS patients improved spontaneously. The results obtained from small numbers of patients in open studies, including our own limited experience in CIDP patients with an IgG or IgM monoclonal protein, indicate that the patients with an IgG, but probably also some patients with a 
IgM monoclonal gammopathy, can improve after IVIg treatment. ${ }^{813-15}$ Whether a gammopathy is a prognostic factor for the chance of improvement after IVIg treatment is at present unknown.

\section{IVIg mechanism of action in inflammatory demyelinating polyneuropathies}

Studies on various auto-immune diseases show that IVIg can interfere with the regulation of the immune-network, either by stimulation, blockage, or down-regulation. Reviews of the clinical use of IVIg, and the various mechanisms of immunomodulation by IVIg have recently been published. ${ }^{25} 26$ Studies on the mechanism of IVIg in CIDP are limited. In vitro studies have shown that IVIg contains anti-idiotypic antibodies that recognise a cross-reactive idiotype on anti-neuroblastoma (NBL) cell line antibodies present in roughly $50 \%$ of patients with GBS or CIDP. $\mathrm{F}(\mathrm{ab})_{2}$ antibodies present in serum from patients that have recovered from GBS can inhibit antiNBL antibody activity in serum from patients with CIDP or with active GBS. ${ }^{27-29}$ These inhibitory antibodies which develop during recovery from GBS are also present in IVIg. Affinity chromatography revealed that these anti-idiotypic antibodies constitute less than $1 \%$ of the total IgG antibodies present in IVIg. ${ }^{30}$ It is, however, not proven that the various antibodies, including anti-NBL antibodies (that are present in GBS and in CIDP patients) have a direct pathogenic role in the process of disease. Whether a large pool of donors is essential for improvement from GBS or CIDP is questionable since we have observed that improvement from CIDP can also occur after intermittent infusions of fresh frozen plasma (FFP) obtained from a limited number (20-28) of donors. ${ }^{9}$ Although the mechanism of action of IVIg in patients with GBS or CIDP is far from clarified, there are arguments obtained from in vitro studies that $\mathrm{V}$ region-dependent interaction may play a role in the regulation of GBS and CIDP.

\section{Conclusion}

Patients with CIDP can improve after IVIg treatment. The proportion of patients that improve after IVIg is difficult to establish and seems to be dependent on the selection of patients. Further studies should focus on prognostic criteria in relation to improvement after IVIg. Studies comparing the effect of IVIg with PE or steroids are eagerly awaited. These studies, however, should not only look for the initial clinical significant improvement, but should also study the necessity of maintenance treatment. Since most patients with a CIDP need treatment for a long period of time, cost-benefit studies need to focus not only on the initial phase of treatment but also on the effects of long-term treatment including the potential side-effects of steroid treatment. We have such a study in progress now. Studies on small numbers of CIDP patients with a monoclonal gammopathy suggest that these patients may respond after IVIg as well. However, controlled studies are urgently required.

The mechanism of action of IVIg in CIDP is far from being completely understood. It seems that immune-regulation via V-region interaction at the antibody level may play a role. Some other mechanisms by which IVIg might interact are the induction of T-suppressor cells, down-regulation of TNF-alpha and other cytokines, interference with Fc-receptors either on nervous tissue or on B cells, and a specific action on $B$ cells that bear the pathogenic idiotype. Further studies on the effect of IVIg in CIDP may reveal important features that can be of help in unravelling the pathogenesis of the inflammatory demyelinating polyneuropathies.

We thank our colleagues for referring patients, and the Prinses Beatrix Fonds and the Kröger Stichting for support.

1 Dyck PJ, Prineas J, Pollard JD. Chronic inflammatory demyelinating polyneuropathy. In: Dyck PJ, Thoma demyelinating polyneuropathy. In: Dyck PJ, Thomas Saunders, 1993:1498-518.

2 Asbury AK, Cornblath DR. Assessment of current diagnostic criteria for Guillain-Barré syndrome. Ann Neurol 1990;27(suppl):S21-4.

3 Hughes RAC, Sanders E, Hall S, Atkinson P, Colchester A, Payan P. Subacute idiopathic demyelinating polyradiculoneuropathy. Arch Neurol 1992;49:612-6.

4 Cornblath DR, Asbury AK, Albers JW, et al. Research criteria for diagnosis of chronic inflammatory demyelinating polyneuropathy (CIDP). Neurology 1991;41:617-8.

5 Dyck PJ, O'Brien PC, Oviatt KF, et al. Prednisone improves chronic inflammatory demyelinating polyradiculoneuropathy more than no treatment. Ann Neurol 1982;11:136-41.

6 Dyck PJ, Daube J, O'Brien, et al. Plasma exchange in chronic inflammatory demyelinating polyradiculoneuropathy. $N$ Engl f Med 1986;314:461-5.

Vermeulen M, Van Doorn PA, Brand A, Strengers PFW, Jennekens FGI, Busch HFM. Intravenous immunoglobulin treatment in patients with chronic inflammatory demyelinating polyneuropathy: a double blind, placebo controlled study. F Neurol Neurosurg Psychiatry 1993; 56:36-9.

8 Dyck PJ. Intravenous immunoglobulin in chronic inflammatory demyelinating polyradiculoneuropathy and in neuropathy associated with IgM monoclonal gammopathy of unknown significance. Neurology 1990;40:327-8.

9 Vermeulen $M$, van der Meché FGA, Speelman JD, et al. Plasma and gamma-globulin infusion in chronic inflammatory demyelinating polyneuropathy. $f$ Neurol $\mathrm{Sci}$ matory demyelinati

10 Curro Dossi B, Tezzon F. High-dose intravenous gammaglobulin for chronic inflammatory demyelinating polyneuropathy. Ital $₹$ Neurol Sci 1987;8:321-6.

11 Faed JM, Day B, Pollock M, et al. High-dose intravenous human immunoglobulin in chronic inflammatory demyelinating polyneuropathy. Neurology 1989;39. $422-5$.

12 Vedanarayanan VV, Kandt RS, Lewis DV, DeLong GR. Chronic inflammatory demyelinating polyradiculoneuropathy of childhood. Treatment with high-dose intravenous immunoglobulin. Neurology 1991;41:828-30.

13 Cornblath DR, Chaudry V, Griffin JW. Treatment of chronic inflammatory demyelinating polyneuropathy with intravenous immunoglobulin. Ann Neurol 1991; with intrave

14 Van Doorn PA, Vermeulen $M$, Brand A, Mulder PGH, Busch HFM. Intravenous immunoglobulin treatment in patients with chronic inflammatory demyelinating patients with chronic inflammatory demyelinating polyneuropathy. Clinical and laboratory characteristics associated

15 Azulay JPh, Pouget J, Pellissier JF, Blin O, Serratrice G Polyradiculonevrites chroniques. Rev Neurol (Paris) 1992;148:752-61

16 Van Doorn PA, Brand A, Strengers PFW, Meulstee J, Vermeulen $M$. High-dose intravenous immunoglobulin treatment in chronic inflammatory demyelinatin polyneuropathy. A double-blind placebo-controlled crossover study. Neurology 1990;40:209-12.

17 Thornton CA, Griggs RC. Plasma exchange and intravenous immunoglobulin treatment of neuromuscular disease. Ann Neurol 1994;35:260-8.

18 Newberger JW, Takahashi M, Beiser AS, et al. A single intravenous infusion of gammaglobulin as compared with four infusions in the treatment of Kawasaki syndrome. N Engl f Med 1991;324:1633-9. 
19 Reinhart WH, Berchtold PE. Effect of high-dose intravenous immunoglobulin therapy on blood rheology. venous immunoglobulin

20 Woodruff RK, Grigg AP, Firkin FC, Smith IL. Fatal thrombotic events during treatment of autoimmune thrombotic events during treatment of autoimmune thrombocytopenia with intravenous im

21 Dyck PJ, Lais AC, Ohta M, et al. Chronic inflammatory demyelinating polyradiculoneuropathy. Mayo Clin Proc 1975;50:621-37.

22 Suarez GA, Kelly JJ. Polyneuropathy associated with monoclonal gammopathy of undetermined significance: further evidence that IgM-MGUS neuropathies are different than IgG-MGUS. Neurology 1993;43:1304-8.

23 Bromberg MB, Feldman EL, Albers JW. Chronic inflammatory demyelinating polyradiculoneuropathy: comparison of patients with and without an associated son of patients with and without an associated
monoclonal gammopathy. Neurology 1992;42:1157-63.

24 Simmons Z, Albers JW, Bromberg MB, Feldman EL. Presentation and initial clinical course in patients with chronic inflammatory demyelinating polyradiculoneuropathy: comparison of patients without and with monoclonal gammopathy. Neurology 1993;43:2202-9.
25 Dwyer JM. Manipulating the immune system with immune globulin. N Engl f Med 1992;326:107-16.

26 Hurez V, Kaveri SV, Kazatchkine MD. Normal polyspecific immunoglobulin G (IVIg) in the treatment of

27 Van Doorn PA, Brand A, Vermeulen M. Anti-neuroblastoma cell line antibodies in inflammatory demyelinating polyneuropathy: inhibition in vitro and in vivo by IV immunoglobulin. Neurology 1988,38.1592-5.

28 Lundkvist I, Van Doorn PA, Vermeulen M, Van Lint $M$, Van Rood J, Brand A. Regulation of autoantibodies in inflammatory demyelinating polyneuropathy: spontaneous and therapeutic. Immunol Rev 1989;110:105-17.

29 Van Doorn PA, Rossi F, Brand A, van Lint M, Vermeulen $M$, Kazatchkine $\mathrm{MD}$. On the mechanism of high-dose intravenous immunoglobulin treatment of patients with chronic inflammatory demyelinating polyneuropathy. ₹ Neuroimmunol 1990;29:57-64.

30 Lundkvist I, Van Doorn PA, Vermeulen M, Brand A. Spontaneous recovery from the Guillain-Barre syndrome is associated with anti-idiotypic antibodies recognizing a cross-reactive idiotype on anti-neuroblastoma cell line antibodies. Clin Immunol Immunopathol 1993;67:192-8. 